Research Paper

\title{
High Expression of Indoleamine 2, 3-Dioxygenase in Adenosquamous Lung Carcinoma Correlates with Favorable Patient Outcome
}

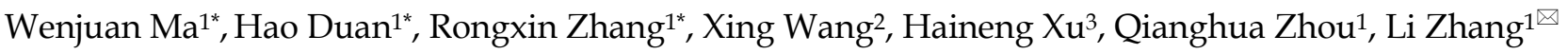 \\ 1. Sun Yat-Sen University Cancer Center, State Key Laboratory of Oncology in South China, Collaborative Innovation Center for Cancer Medicine, \\ Guangzhou, 510060, China \\ 2. Tongji Medical College, Huazhong University of Science and Technology, Wuhan, 430030, China \\ 3. Ovarian Cancer Research Center, Division of Gynecology Oncology, Department of Obstetrics and Gynecology, University of Pennsylvania, Philadelphia, \\ 19104, USA \\ *These authors contributed equally to this work. \\ $\triangle$ Corresponding author: Li Zhang. Email: zhangli@sysucc.org.cn. Address: Sun Yat-sen University Cancer Center, State Key Laboratory of Oncology in South \\ China, Collaborative Innovation Center for Cancer Medicine, 651 Dongfeng East Road Guangzhou, Guangdong, 510060, China \\ (c) Ivyspring International Publisher. This is an open access article distributed under the terms of the Creative Commons Attribution (CC BY-NC) license \\ (https://creativecommons.org/licenses/by-nc/4.0/). See http://ivyspring.com/terms for full terms and conditions.
}

Received: 2018.05.27; Accepted: 2018.10.03; Published: 2019.01.01

\begin{abstract}
Background: Indoleamine 2,3-dioxygenase (IDO), an enzyme involved in tryptophan (Trp) metabolism, is generally considered to be an immunosuppressive molecule. The prognostic role of IDO expression in tumor has not been well studied in non-small cell lung cancer (NSCLC) and even not been reported in adenosquamous lung carcinoma (AdSqLC). Herein, the aim of this study is to investigate the prognostic significance of IDO expression in patients with AdSqLC.

Patients and Methods: We conducted immunohistochemical analyses of IDO expression, as well as CD3 and CD8 expression, in 183 primary tumor tissue samples from patients with AdSqLC treated at our institution between July 1999 and September 2014. Patients' clinicopathological characteristics were retrospectively reviewed. Survival analysis was performed in the entire cohort of patients and those who received radical resection, respectively.

Results: IDO was expressed in 146 (79.8\%) tumor samples. A higher level of IDO expression was significantly associated with increased CD8+ tumor-infiltrating lymphocytes (TILs) in tumor tissues $(P<0.001)$. Surprisingly, overall survival $(O S)$ was significantly better for patients with high IDO expression (hazard ratio $(H R)=0.505$; confidence interval $(C l)=0.329-0.775 ; P=0.002$ ) for the entire cohort. In patients who were unable to be treated with radical resection, IDO expression had no effect on $\mathrm{OS}(\mathrm{P}=0.598)$. In contrast, a significant, independent association between high expression of IDO and better OS $(\mathrm{HR}=0.469 ; \mathrm{Cl}=0.290-0.758 ; \mathrm{P}=0.002)$ was identified in patients who received radical resection.

Conclusions: IDO is expressed in most AdSqLC tissues, with a higher level of IDO expression associated with an occurrence of CD8+ TILs. Moreover, IDO expression in tumor promises to serve as a strongly independent favorable prognostic factor, particularly in patients who received radical resection.
\end{abstract}

Key words: Indoleamine 2,3-dioxygenase, lung cancer, radical resection, prognosis

\section{Introduction}

Lung cancer is one of the most common malignant tumors and is the leading cause of cancer mortality in both developed and developing countries
[1]. Non-small cell lung cancer (NSCLC) accounts for almost $85 \%$ of lung cancer cases. Adenosquamous lung carcinoma (AdSqLC) is a rare subtype of NSCLC 
that accounts for $2-4 \%$ of lung cancer[2]. This type is characterized by comprising both squamous cell carcinoma and adenocarcinoma components at least $10 \%$ each of the total tumor volume.

Indoleamine 2,3-dioxygenase (IDO, also referred to as IDO1), is a cytosolic, heme-containing enzyme consisting of 403 amino acids and encoded by the INDO gene on human chromosome $8 \mathrm{p} 22$. The basic function of IDO is the catabolism of tryptophan, an essential amino acid, in the kynurenine (Kyn) pathway. IDO leads to the degradation of tryptophan, which is necessary to maintain proper Kyn concentrations and for other vital cellular functions[3]. In normal human tissues, IDO is expressed by endothelial cells in the placenta and lung, by epithelial cells in the female genital tract, and by mature dendritic cells in lymphoid tissues[4]. For human cancers, the highest proportions of IDOexpressing cells were observed in endometrial and cervical carcinomas, followed by kidney, lung, and colon cancers. Initially, IDO was thought to be a tumor suppressor[5]. The expression of IDO induced by interferon gamma (IFN- $\gamma$ ) presented an antiproliferative effect through tryptophan (Trp) deprivation[6, 7]. This view has been changed since the discovery of the role of IDO in mediating immune privilege during pregnancy[8]. IDO activity was found to be involved in acquired immune tolerance, including inhibition of T-cell activation and induction of regulatory $\mathrm{T}$ cells (Tregs) activation[9-11], which can allow tumor cells to escape or evade immunosurveillance[12].

The prognostic role of IDO expression and/or activity has been reported in several human cancers. High expression and/or activity of IDO has generally been reported to correlate with a worse prognosis, increased disease stage, presence of metastatic disease, decreased tumor-infiltrating lymphocytes, and increased FoxP3p Tregs[13-16]. However, there are still some exceptions. IDO expression in tumor tissues could serve as a favorable factor for patient survival in basal-like breast carcinoma, hepatocellular carcinoma and renal cell carcinoma[17-19]. In NSCLC, the large majority of studies are mainly focused on the serum $\mathrm{Kyn} / \mathrm{Trp}$ ratio, which is used as a surrogate indicator of IDO activity. It has been reported that an increased serum Kyn/Trp ratio is associated with more advanced stages, chemotherapy resistance and decreased survival times after chemo-radiation therapy[20-23]. However, the patients in these previous studies were mainly inoperable/unresectable. On the other hand, only one study tested the prognostic role of IDO expression in NSCLC tumor tissue[24]. In this study, reported by S. Astigiano et al., IDO was expressed not by tumor cells but by eosinophil granulocyte infiltrating the peritumoral stroma. Patients with a greater concentration of IDO-positve eosinophil granulocytes tended to have a shorter survival. However, the reliability of results in the previous study were restricted by its small sample size. Only 25 tumor samples were analyzed and 17 patients with adequate follow-up were further involved in a simplified survival analysis.

Given that the prognostic role of IDO expression in tumor has not been well studied in NSCLC and even not been reported in AdSqLC, the aim of the present study is to investigate the association of IDO expression in tumor with clinicopathological features and outcomes in patients with AdSqLC.

\section{Results}

\section{IDO, CD3, and CD8 expression in tumor tissues}

Figure 1 shows the typical junction area of glandular and squamous components of tumor specimens involved in this study. IDO was mainly expressed by tumor cells accompanied by sporadic IDO-positive stromal cells and blood vessels in both adenocarcinoma and squamous carcinoma tissues (Figure 2). IDO positive stains were observed in 146 $(79.8 \%)$ tumor samples. These 146 samples included $12(6.6 \%), 70(38.3 \%)$, and $64(35.0 \%)$ samples that were weakly, moderately, and strongly positive, respectively. Most samples contained less than $50 \%$ of positive cells. The CD3+ TILs (Figure 3A) were present in $118(64.5 \%)$ samples and $65(35.5 \%)$ patients had occurrences of CD8+ TILs (Figure 3B) in their tumor tissues.

\section{Association of IDO expression with clinicopathological characteristics}

The median age of the patients was 58.0 years (range, 30.0-80.0 years). Median and mean survival times were 43.0 and 82.6 months, respectively. The 1-, 3 -, and 5-year survival rates were $80.5 \%, 53.2 \%, 44.7 \%$, respectively. Table 1 shows the distribution of patients' clinicopathologic characteristics according to the levels of IDO expression. Of the examined characteristics, only CD8+ TILs $(\mathrm{P}<0.001)$ was significantly associated with IDO expression, with a higher level of IDO expression associated with an occurrence of CD8+ TILs in tumor tissues. We did not observe a relationship between IDO expression and other factors including age, sex, T status, N status, M status, TNM staging, smoking, CD3+ TILs and treatments. 

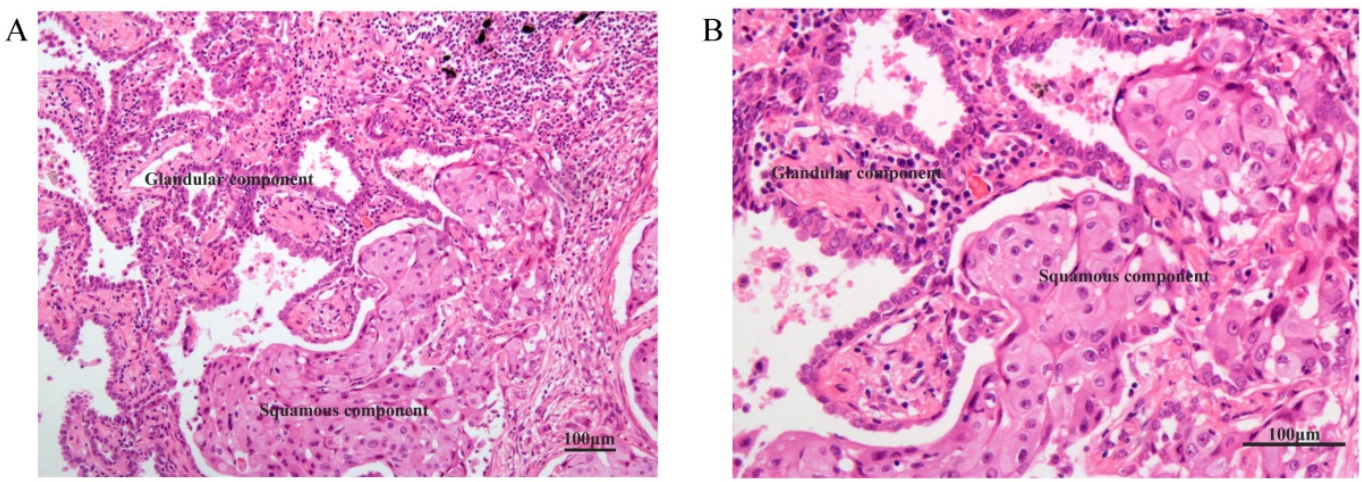

Figure 1. HE staining of the typical junction area of glandular and squamous components of AdSqLC. The top right area is the glandular component, and the bottom right area is the squamous component. A: 100x. B: 200x.
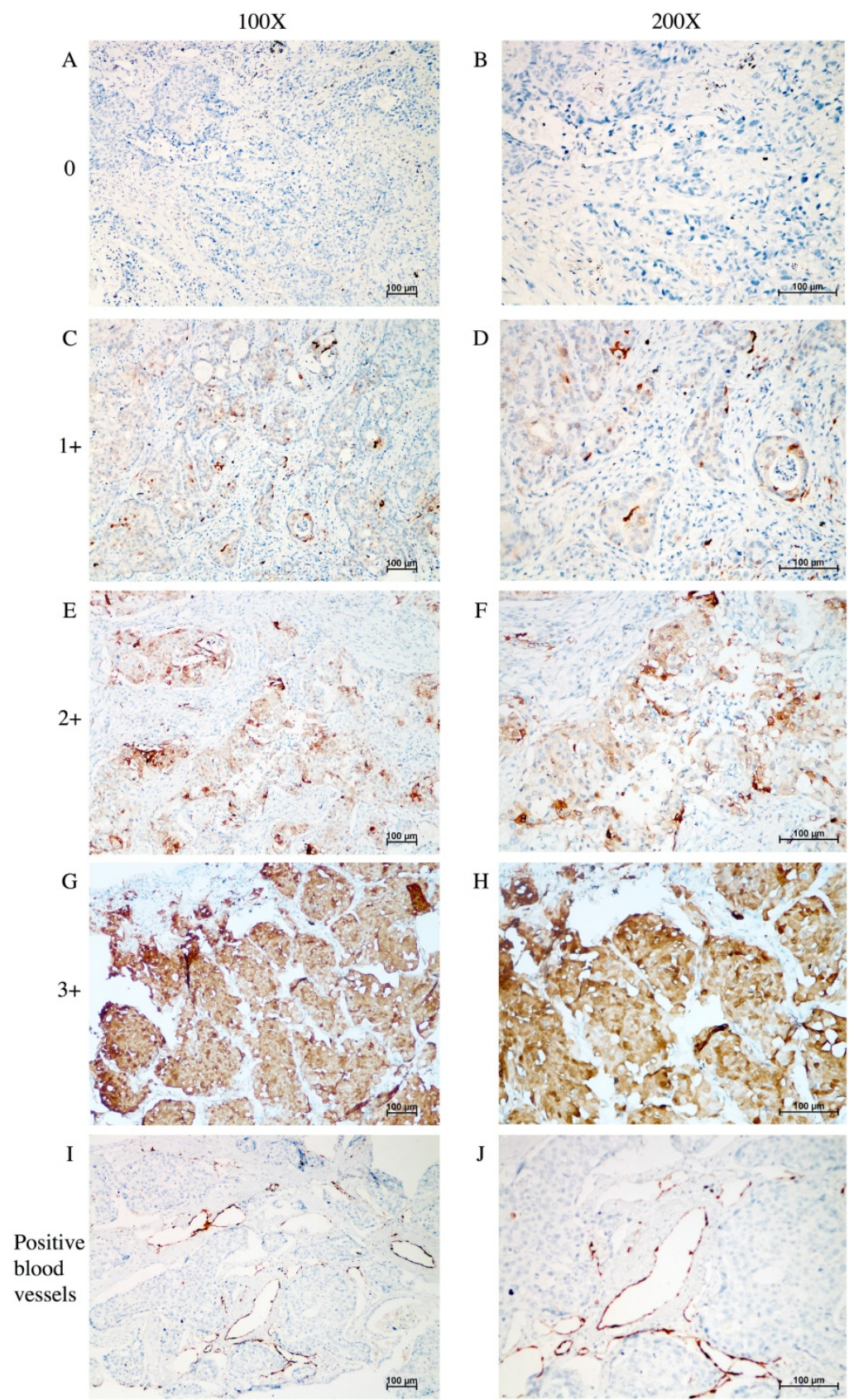

Figure 2. IHC analysis of IDO expression in tumor tissue samples taken from patients with AdSqLC. A-H: representative image of IDO expression in tumor cells and sporadic stromal cells, no positive $=0$, weak positive $=1+$, moderate positive $=2+$, and strong positive $=3+$. I-J: representative image of IDO-positive blood vessels. 


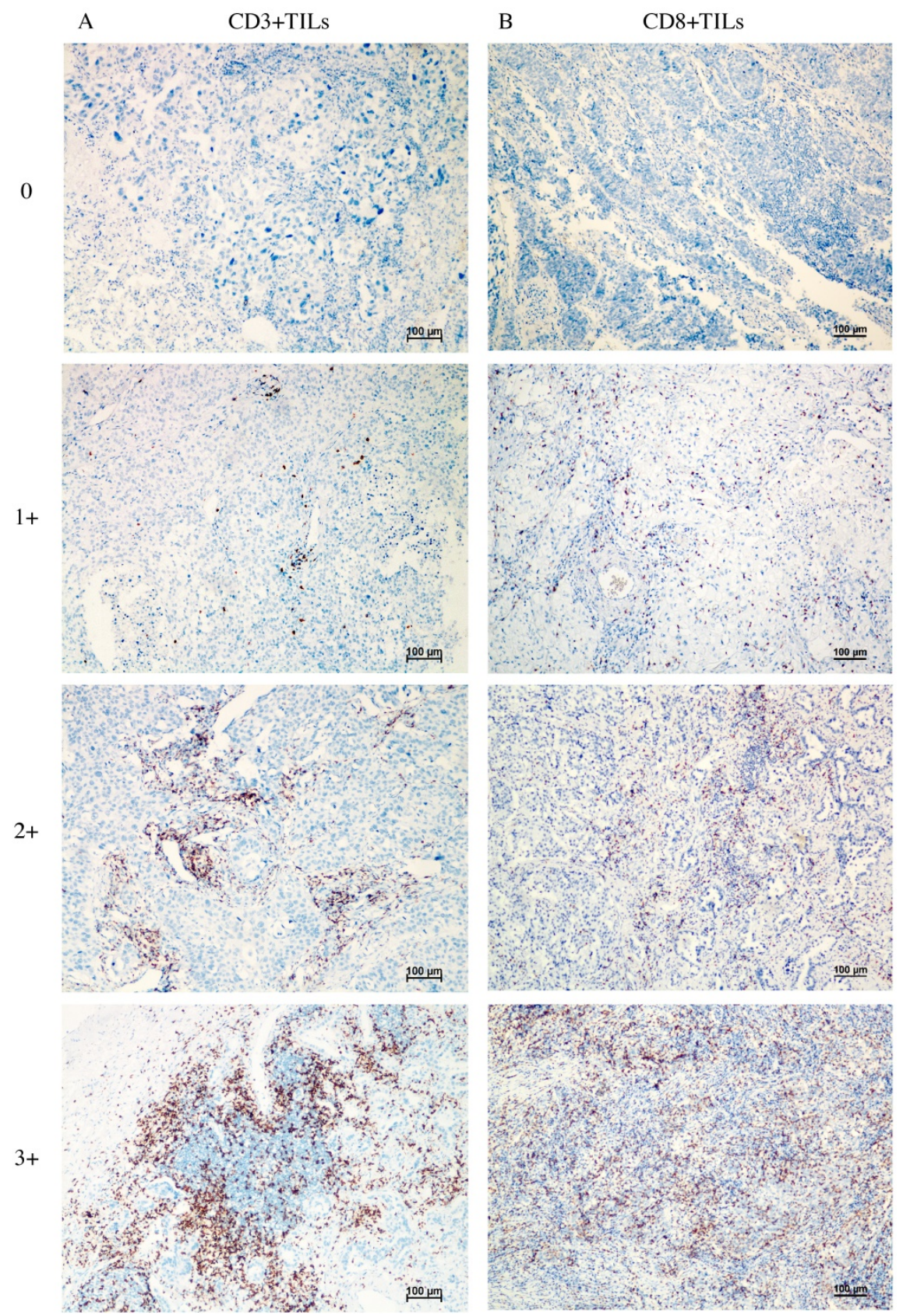

Figure 3. Representative image of CD3+ TILs and CD8 + TILs in tumor tissues, no occurrence $=0$, sporadic occurrence $=1+$, moderate occurrence $=2+$, abundant occurrence $=3+$. A: representative image of CD3+ TILs. B: representative image of CD8+ TILs.

\section{Association of IDO expression with patient prognosis}

Not surprisingly, T status, $\mathrm{N}$ status and TNM staging were negatively associated with OS (all $\mathrm{P}<0.05$ ) in Kaplan-Meier analysis (Table 2). However, the expression of IDO in tumor tissues significantly correlated with overall survival $(\mathrm{P}<0.001)$ (Figure $4 \mathrm{~A})$. The mean and median OS values of patients with high IDO expression were 113.5 and 163 months, respectively, while the values of patients with low IDO expression were 62.6 and 32 months, respectively. Age, sex, smoking status, M status, CD3+ TILs, CD8+
TILs, and treatments were not associated with overall survival.

To assess the statistical independence of the prognostic role of IDO expression, we further performed the multivariate Cox regression analysis (Table 3). Due to the co-linearity of T status, N status and TNM staging, we put IDO and TNM staging only into the multivariable analysis given that TNM staging is a universal indicator of survival. Both IDO expression $(\mathrm{HR}=0.505 ; \mathrm{CI}=0.329-0.775 ; \mathrm{P}=0.002)$ and TNM staging $(\mathrm{HR}=1.358 ; \mathrm{CI}=1.118-1.648 ; \mathrm{P}=0.002)$ were independently associated with OS. 
Table 1. Patients' clinicopathologic features compared with IDO expression.

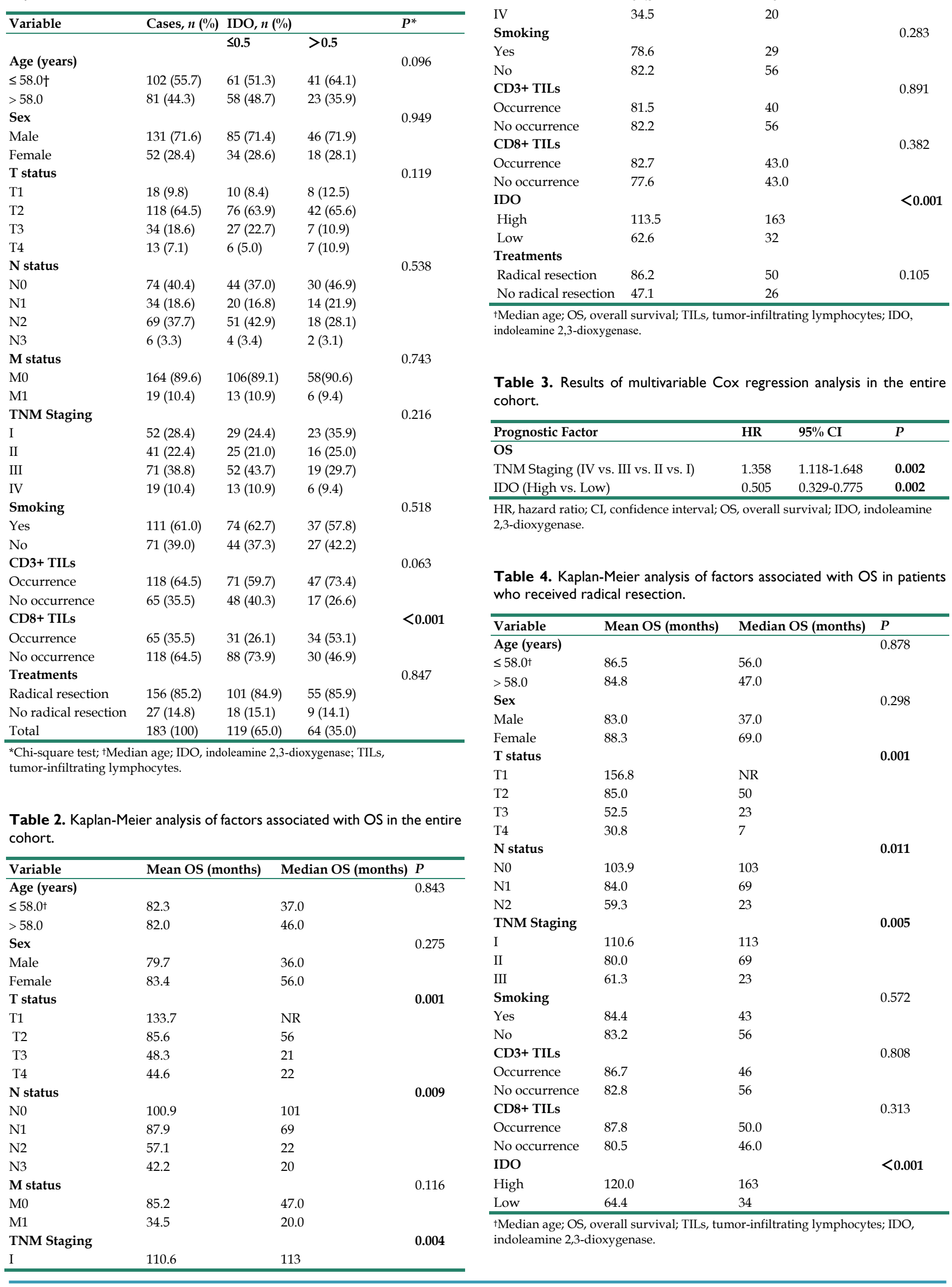



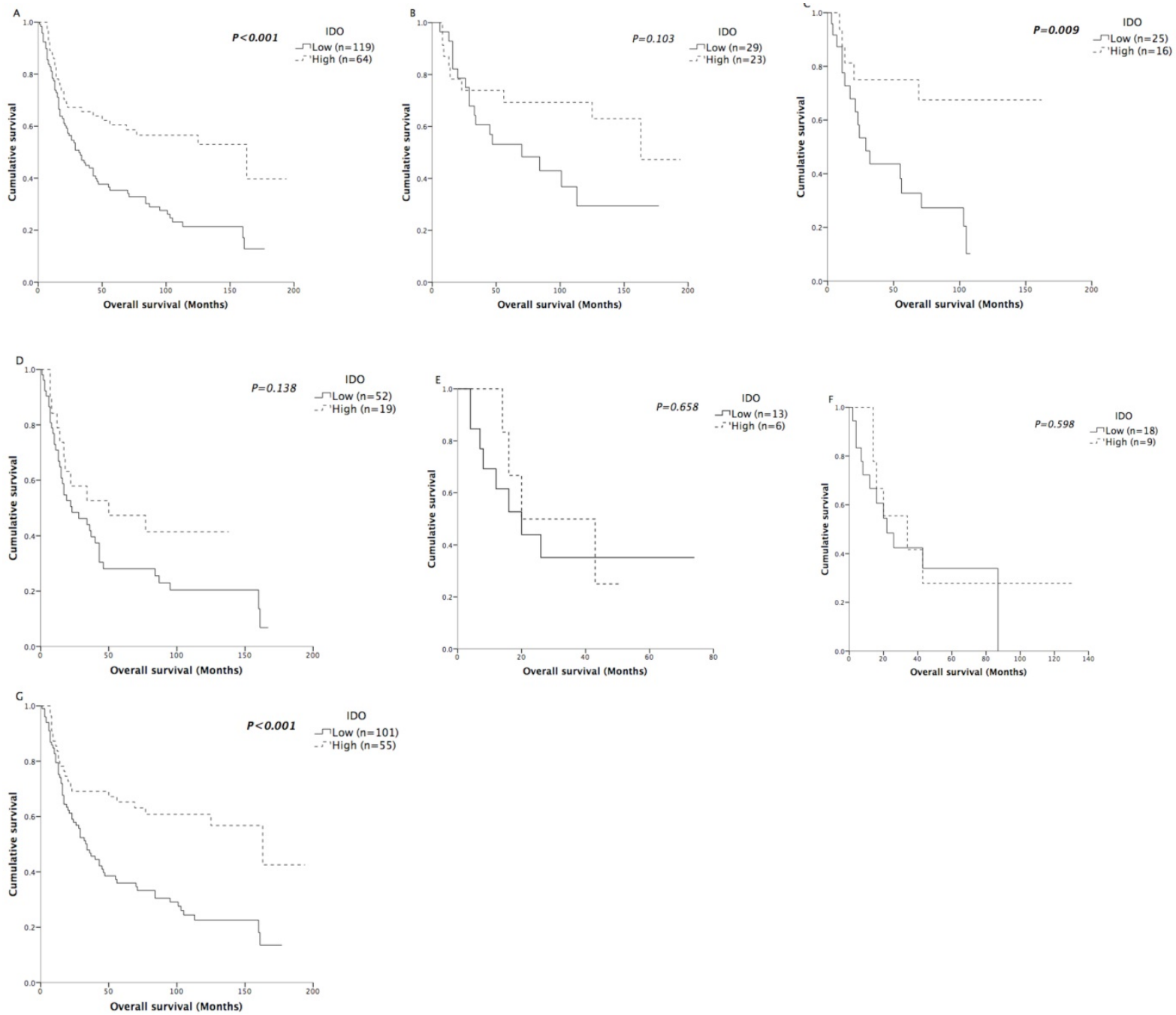

Figure 4. Kaplan-Meier analysis of OS according to IDO expression. Differences between curves were assessed by the log-rank test. A: patients in the entire cohort $(n=183)$. B: patients with stage I disease $(n=52)$. C: patients with stage II disease $(n=41)$. D: patients with stage III disease $(n=71)$. E: patients with stage IV disease $(n=19)$. F: patients treated without radical resection $(n=27)$. G: patients treated with radical resection $(n=156)$.

We also further assessed the prognostic effect of IDO after stratification by TNM staging. In all stages except stage IV disease, the survival curves showed obvious trends of better survival in patients with high IDO expression (Figure 4B-4E). Although the survival differences in stage I and stage III patients did not reach statistical significance, we believed it was due to the limited number of patients in each stage.

\section{Association between IDO expression and prognosis in patients who received radical resection}

In previous studies, the prognostic role of IDO activity, represented by the serum Kyn/Trp ratio, was mainly reported in NSCLC patients who were inoperable/unresectable and received chemotherapy or chemoradiation as their primary treatment [20-23]. Because IDO expression had a prognostic role in all stages except stage IV disease in our patient cohort, we speculated that IDO expression would be a good prognostic factor for patients who received radical resection. Therefore, the predictive significance of IDO expression on OS was further assessed according to treatments. Among the 183 patients, 156 of them received radical resection. As expected, the IDO expression of patients who were unable to be treated with radical resection was not correlated with OS $(\mathrm{P}=0.598)$ (Figure $4 \mathrm{~F})$. For patients who received radical resection, the mean OS of the high-expression patients was 55.6 months longer compared with that of the low- expression patients $(\mathrm{P}<0.001)$ (Figure $4 \mathrm{G})$. Other clinicopathological characteristics were also analyzed by Kaplan-Meier analysis (Table 4) in patients who received radical resection. Similarly, $\mathrm{T}$ status, $\mathrm{N}$ status and TNM staging were negatively associated with OS (all $\mathrm{P}<0.05$ ). In multivariate analysis (Table 5), both IDO expression (HR=0.469; $\mathrm{CI}=0.290-0.758 ; \mathrm{P}=0.002$ ) and TNM staging ( $\mathrm{HR}=$ 1.380; $\mathrm{CI}=1.083-1.759 ; \mathrm{P}=0.009$ ) were independently associated with OS. 
Table 5. Results of multivariable Cox regression analysis in patients who received radical resection.

\begin{tabular}{lccc}
\hline Prognostic Factor & HR & $\mathbf{9 5 \%}$ CI & $\boldsymbol{P}$ \\
\hline OS & & & \\
TNM Staging (IV vs. III vs. II vs. I) & 1.380 & $1.083-1.759$ & $\mathbf{0 . 0 0 9}$ \\
IDO (High vs. Low) & 0.469 & $0.290-0.758$ & $\mathbf{0 . 0 0 2}$ \\
\hline
\end{tabular}

HR, Hazard ratio; CI, confidence interval; OS, overall survival; IDO, indoleamine 2,3-dioxygenase.

\section{Discussion}

Previous studies have reported the prognostic role of IDO expression and/or activity in a number of human cancers[28]. Although IDO expression and/or activity were usually associated with a worse prognosis, our results suggested that the IDO expression in tumor was a favorable factor for OS in AdSqLC. In previous studies, IDO activity, represented by the serum Kyn/Trp ratio, portended worse OS in NSCLC patients who were treated with chemotherapy and/or chemoradiation[20-23]. Unfortunately, the corresponding serums of patients included in this study were unavailable. However, the relationship between serum Kyn/Trp ratio and IDO expression in the tumor has been examined in other studies. In cervical cancer, tumor IDO expression was associated with an increased serum Kyn/Trp ratio[29]. In colorectal carcinoma, both serum Kyn/Trp ratio and IDO expression correlated with the presence of lymphatic invasion and lymph node metastasis[30]. Therefore, we believe that serum Kyn/Trp ratio and IDO expression are comparable. One reason may account for the opposite findings in the previous studies compared with our results. The patients in the previous studies were mainly inoperable/unresectable and received chemotherapy or chemoradiation as their primary treatments, while the large majority of patients in the present study underwent radical surgery, which may also account for the relatively high patients' survival rates in the present study. Indeed, the better survival in patients with high IDO expression was only observed in those who were treated with radical resection. In line with our results, IDO expression in basal-like breast carcinoma, hepatocellular carcinoma, renal cell carcinoma and cervical cancer was found to correlate with prolonged survival[17-19, 29], which suggests that IDO could slow tumor progression as well.

IDO expression has been observed in the majority (58\%) of human tumors[4]. In NSCLC, it was reported that IDO was expressed by tumor cells, blood vessels, and stromal cells[4]. S. Astigiano et al. investigated IDO expression in 25 NSCLC patients and found IDO was expressed, not by tumor cells, but by normal eosinophil granulocytes infiltrating the peritumoral stroma[24]. Meanwhile, they also found that the expression of IDO in eosinophil granulocytes was negatively associated with patient survival. However, the reliability of these results might be compromised by the small sample size and the improper survival analysis. Due to the limited sample size, only 17 patients, including 15 adenocarcinoma and 2 squamous cell carcinoma patients, with complete staging and adequate follow-up were selected to analyze the association between IDO-expressing eosinophil granulocytes and patient survival. Nevertheless, instead of Kaplan-Meier analysis and Cox proportional hazards model, Spearman correlation analysis was used for the survival analysis. In the present study, the majority of IDO-positive cells were tumor cells accompanied by sporadic stromal cells and blood vessels, which was consistent with previous study[4]. We conducted immunohistochemical analyses of $183 \mathrm{AdSqLC}$ tumor tissues that were composed of at least $10 \%$ each of squamous cell carcinoma and adenocarcinoma cells. Given that AdSqLC is an independent subtype of NSCLC, we scored the IDO expression of each sample in its entirety. Although it is reasonable to believe that IDO would also serve as a favorable prognostic factor in both lung adenocarcinoma and squamous cell lung carcinoma, studies for these two main subtypes of NSCLC are still warranted to confirm our findings.

In addition, we found that IDO expression was not associated with any of the examined clinicopathological characteristics except CD8+ TILs. A higher occurrence of CD8+ TILs tended to accompany a higher expression of IDO in tumor tissues, which was also reported in cervical cancer[29]. Intriguingly, this would be in line with the observation that activated T-cells are able to induce IDO expression in tumor cells through IFN- $\gamma[19,31]$. Meanwhile, K. A. Schalper et al. also reported that IDO expression was associated with increased TILs and IFN- $\gamma$ stimulation in lung carcinomas[32]. However, CD8+ TILs were not associated with patient survival in our study, which indicated that the antitumor effect of IDO was independent of CD8+ TILs. Given IDO is an enzyme involved in the metabolism of the essential amino acid tryptophan, the favorable influence of IDO expression in tumors may be due to inhibition of tumor cell proliferation by depleting tryptophan[5-7], or induction of tumor cells apoptosis by forming toxic Typmetabolites[33]. Furthermore, T. Ishio et al. reported that the cytotoxicity of peripheral blood mononuclear cell (PBMC) against certain hepatocellular carcinoma cell lines was significantly reduced by treating tumor cells with an IDO inhibitor[18], which suggested that IDO could enhance tumor sensitivity to anticancer immune reactions. 
In the past few years, gene therapy and immunotherapy are emerging to be the new promising strategies for cancer therapy[34, 35]. Recently, immunotherapy using antibodies against PD-1 or PD-L1 has shifted the treatment paradigm for NSCLC. Despite long-lasting remissions in patients who respond, the low response rate limits the application of current immuno-therapeutic strategies[36]. Therefore, there is a strong interest in identifying new immune escape mechanisms, predictive biomarkers, and new therapeutic targets. Although IDO is generally considered as an immunosuppressive molecule that can facilitate immune escape/evasion by the tumor[12], our results, along with some previous studies, indicate that the role of IDO in human cancers may be more complex than anticipated[5-7, 17-19, 29]. In certain cancers, the expression of IDO may even benefit patient survival, which may facilitate future clinical trial enrollment and individualized treatments.

The significance of the present study was limited by its retrospective design. In addition, only 27 patients treated without radical resection were included in the analyses. As a result, the small cohort may account for the convergent survival curves in these patients.

In conclusion, this study showed that IDO is expressed in most AdSqLC tissues, with a higher level of IDO expression associated with an occurrence of CD8+ TILs. Moreover, IDO expression represents a favorable prognostic factor, particularly in patients who received radical resection.

\section{Abbreviations}

AdSqLC: adenosquamous lung carcinoma; CI: confidence interval; CT: computed tomography; HR: hazard ratio; I: intensity; IDO: indoleamine 2,3dioxygenase; IFN- $\gamma$ : interferon gamma; IHC: immunohistochemistry; Kyn: kynurenine; M: distant metastasis; MRI: magnetic resonance imaging; $\mathrm{N}$ : lymph node; NSCLC: non-small cell lung cancer; OS: overall survival; PBMC: peripheral blood mononuclear cell; Prop: proportion; ROC: receiver operating characteristic; T: tumor; TILs: tumor-infiltrating lymphocytes; Trp: tryptophan.

\section{Patients and Methods}

\section{Patients}

We conducted a retrospective review of the records of 235 patients with histologically confirmed primary AdSqLC who were treated at Sun Yat-Sen University Cancer Center between July 1999 and September 2014. The exclusion criteria were as follows: (1) patients whose tumor tissues were unavailable; (2) patients for whom follow-up data were not detailed or complete; (3) patients who died from non-tumor causes. After applying the exclusion criteria, 183 eligible patients were included in the study. A single pathologist conducted a second review of pathologic specimens. Clinicopathological data including patient age, sex, smoking history, tumor $(\mathrm{T})$ status, lymph node $(\mathrm{N})$ status, distant metastasis (M) status, TNM staging and treatments were collected from medical records. Patients were treated according to the National Comprehensive Cancer Network (NCCN) guidelines. Generally, inoperable diseases were treated by chemoradiotherapy or chemotherapy after biopsy. For patients eligible for surgical excision, standard radical resections were performed. After surgical evaluation, eligible patients received adjuvant chemotherapy. The occurrence of tumor-infiltrating lymphocytes (TILs) in tumor tissues was also analyzed along with the expression of IDO by immunohistochemical (IHC) staining. The overall survival (OS) was defined as the time between diagnosis of AdSqLC and cancer-related death, and patients without an event were censored at the end of the follow-up. Patients were staged according to the TNM classification of the American Joint Committee on Cancer Staging Manual, 8th Edition[25]. This study was approved by the Medical Ethics Committee of Sun Yat-Sen University Cancer Center.

\section{Follow-up}

The follow-up intervals varied according to the condition of each patient. For patients seen in the clinic, we routinely recorded the patient's medical history and conducted a physical examination, contrast-enhanced computed tomography (CT) of the thorax and upper abdomen, as well as brain magnetic resonance imaging (MRI). The last date of follow-up was in October 2017 and included verification of clinical attendance records and direct telecommunication with the patient or the family. During the course of follow-up, 111 patients $(60.7 \%)$ died of lung cancer-related causes.

\section{IHC staining}

Formalin-fixed, paraffin-embedded tumor specimens were sectioned at $5 \mu \mathrm{m}$. For each tumor specimen, ten slices of tumor tissue were sectioned consecutively. The first, fifth and tenth slices were used for IDO staining. The second and third slices were used for CD3 and CD8 staining, respectively. After being incubated at $55^{\circ} \mathrm{C}$ for 3 hours, the samples were deparaffinized in xylene and rehydrated in a series of graded ethanol. Then, the samples were incubated with 3\% hydrogen peroxide for 10-15 mins to block endogenous peroxidase activity. For antigen 
retrieval, the sections were microwaved for 30 minutes in a $0.01 \mathrm{M}$ sodium citrate buffer ( $\mathrm{pH} 6.0$ ), and then pre-incubated in 5\% normal goat serum for 1 hour to prevent nonspecific staining. The sections were incubated with the primary rabbit anti-human IDO monoclonal antibody (working dilution 1:100, Cell Signaling Technology, Danvers, MA, USA, \#86630), rabbit anti-human CD3 monoclonal (working dilution 1:50, Beijing Golden Bridge Biotechnology, China, \#ZA-0503), and mouse anti-human CD8 monoclonal (working dilution 1:100, Beijing Golden Bridge Biotechnology, China, \#ZM-0032) overnight at $4^{\circ} \mathrm{C}$. Subsequently, the samples were incubated with secondary antibodies and $\mathrm{ABC}$ reagent (VECTOR LABORATORIES, Burlingame, CA, USA, \#PK-7200) at room temperature for 30 minutes. Immunoperoxidase staining was performed using an ImmPACT DAB Peroxidase (HRP) Substrate (VECTOR LABORATORIES, Burlingame, CA, USA, \#SK-4105) according to the manufacturer's recommendations. All stained slides were scored independently by two experienced pathologists who were blinded to the patients' identity and clinical status.

\section{Analysis of IHC staining}

IDO expression was scored using the method described in our previous studies[26]. The intensity (I) and proportion (Prop) of staining in each specimen were recorded individually. Scores were calculated as follows: score $=\mathrm{I} \times$ Prop, where $\mathrm{I}$ is the intensity of staining defined as no $=0$, weak $=1$, moderate $=2$, and strong $=3$, and Prop is the fraction of positive cells expressed as a percentage $(0 \%$ to $100 \%)$. The final expression score of IDO is expressed as the average score of three slices per tumor. TILs were assessed by a semi-quantitative estimation of the occurrence of CD3-positive/CD8-positive (CD3+/ CD8+) cells and was scored as no occurrence $=0$, sporadic occurrence $=1$, moderate occurrence $=2$, abundant occurrence $=$ 3[27]. The cut-off point of IDO was defined as 0.5 based on the Youden index of receiver operating characteristic (ROC) curve (Figure 5), which discriminated between survival and death; the area under the curve was 0.640 , and the sensitivity and specificity were $48.6 \%$ and $73.9 \%$, respectively, at the cutoff point. High- and low-expression patients were defined as those of samples with final scores $>0.5$ or $\leq 0.5$, respectively.

\section{Statistical analysis}

The SPSS software package (version 20.0, IBM, USA) was used for the statistical analysis. The correlations between IDO expression and clinicopathologic characteristics, as well as CD3 and CD8 expression, were analyzed using the $\chi^{2}$ test. Survival curves were generated using the Kaplan-Meier method, and differences between curves were assessed by the log-rank test. All significant parameters identified by Kaplan-Meier analysis were evaluated by multivariate analysis using the Cox proportional hazards model. The strength of the association between predictors and survival was assessed by hazard ratios (HRs) with $95 \%$ confidence intervals (CIs). Significance was determined by a two-tailed Student's t-test and a $\mathrm{P}<0.05$ was considered statistically significant.

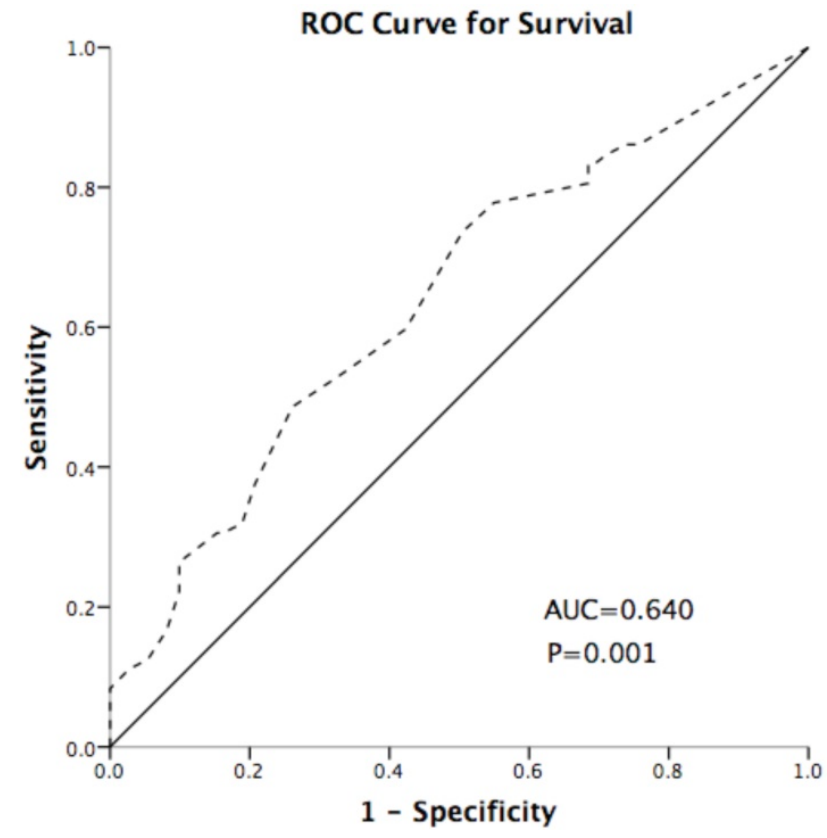

Figure 5. Receiver operating characteristic (ROC) curve for discriminating survival and death using IDO expression. The area under the curve (AUC) value is 0.640 . The sensitivity and specificity were $48.6 \%$ and $73.9 \%$ at the cutoff point. The dash line represents the coordinated points of the ROC curve. The solid line represents the ROC curve diagonal reference line.

\section{Acknowledgments}

This work was supported by National Key R\&D Program of China (grant number: 2016YFC0905500, 2016YFC0905503), Chinese National Natural Science Foundation project (grant number: 81372502), Science and Technology Program of Guangzhou (grant number: 201607020031) and Science and Technology Program of Guangzhou (grant number: 2014000000012). We thank editors at American Journal Experts for professional English improvement. We thank editors at American Journal Experts for professional English improvement.

\section{Competing Interests}

The authors have declared that no competing interest exists. 


\section{References}

1. Torre LA, Bray F, Siegel RL, Ferlay J, Lortet-Tieulent J, Jemal A. Global cancer statistics, 2012. CA Cancer J Clin. 2015; 65: 87-108.

2. Ruffini E, Rena O, Oliaro A, Filosso PL, Bongiovanni M, Arslanian A, et al. Lung tumors with mixed histologic pattern. Clinico-pathologic characteristics and prognostic significance. Eur J Cardiothorac Surg. 2002; 22: 701-7.

3. Takikawa O, Yoshida R, Kido R, Hayaishi O. Tryptophan degradation in mice initiated by indoleamine 2,3-dioxygenase. J Biol Chem. 1986; 261: 3648-53.

4. Theate I, van Baren N, Pilotte L, Moulin P, Larrieu P, Renauld JC, et al. Extensive profiling of the expression of the indoleamine 2,3-dioxygenase 1 protein in normal and tumoral human tissues. Cancer Immunol Res. 2015; 3 : $161-72$.

5. Yoshida R, Park SW, Yasui H, Takikawa O. Tryptophan degradation in transplanted tumor cells undergoing rejection. J Immunol. 1988; 141: 2819-23.

6. Burke F, Knowles RG, East N, Balkwill FR. The role of indoleamine 2,3-dioxygenase in the anti-tumour activity of human interferon-gamma in vivo. Int J Cancer. 1995; 60: 115-22.

7. Ozaki Y, Edelstein MP, Duch DS. Induction of indoleamine 2,3-dioxygenase: a mechanism of the antitumor activity of interferon gamma. Proc Natl Acad Sci U S A. 1988; 85: $1242-6$

8. Munn DH, Zhou M, Attwood JT, Bondarev I, Conway SJ, Marshall B, et al. Prevention of allogeneic fetal rejection by tryptophan catabolism. Science. 1998; 281: 1191-3.

9. Stevens EA, Mezrich JD, Bradfield CA. The aryl hydrocarbon receptor: a perspective on potential roles in the immune system. Immunology. 2009; 127: 299-311.

10. Nguyen NT, Kimura A, Nakahama T, Chinen I, Masuda K, Nohara K, et al. Aryl hydrocarbon receptor negatively regulates dendritic cell immunogenicity via a kynurenine-dependent mechanism. Proc Natl Acad Sci U S A. 2010; 107: 19961-6.

11. Mezrich JD, Fechner JH, Zhang X, Johnson BP, Burlingham WJ, Bradfield CA. An interaction between kynurenine and the aryl hydrocarbon receptor can generate regulatory T cells. J Immunol. 2010; 185: 3190-8.

12. Muller AJ, DuHadaway JB, Donover PS, Sutanto-Ward E, Prendergast GC. Inhibition of indoleamine 2,3-dioxygenase, an immunoregulatory target of the cancer suppression gene Bin1, potentiates cancer chemotherapy. Nat Med. 2005; 11: 312-9.

13. Yu J, Sun J, Wang SE, Li H, Cao S, Cong Y, et al. Upregulated expression of indoleamine 2, 3-dioxygenase in primary breast cancer correlates with increase of infiltrated regulatory $\mathrm{T}$ cells in situ and lymph node metastasis. Clin Dev Immunol. 2011; 2011: 469135

14. Brandacher G, Perathoner A, Ladurner R, Schneeberger S, Obrist P, Winkler C, et al. Prognostic value of indoleamine 2,3-dioxygenase expression in colorectal cancer: effect on tumor-infiltrating T cells. Clin Cancer Res. 2006; 12: 1144-51.

15. Ino K, Yoshida N, Kajiyama H, Shibata K, Yamamoto E, Kidokoro K, et al. Indoleamine 2,3-dioxygenase is a novel prognostic indicator for endometrial cancer. Br J Cancer. 2006; 95: 1555-61.

16. Ino K, Yamamoto E, Shibata K, Kajiyama H, Yoshida N, Terauchi M, et al. Inverse correlation between tumoral indoleamine 2,3-dioxygenase expression and tumor-infiltrating lymphocytes in endometrial cancer: its association with disease progression and survival. Clin Cancer Res. 2008; 14: 2310-7.

17. Jacquemier J, Bertucci F, Finetti P, Esterni B, Charafe-Jauffret E, Thibult ML, et al. High expression of indoleamine 2,3-dioxygenase in the tumour is associated with medullary features and favourable outcome in basal-like breast carcinoma. Int J Cancer. 2012; 130: 96-104.

18. Ishio T, Goto S, Tahara K, Tone S, Kawano K, Kitano S. Immunoactivative role of indoleamine 2,3-dioxygenase in human hepatocellular carcinoma. J Gastroenterol Hepatol. 2004; 19: 319-26.

19. Riesenberg R, Weiler C, Spring O, Eder M, Buchner A, Popp T, et al. Expression of indoleamine 2,3-dioxygenase in tumor endothelial cells correlates with long-term survival of patients with renal cell carcinoma. Clin Cancer Res. 2007; 13: 6993-7002.

20. Suzuki Y, Suda T, Furuhashi K, Suzuki M, Fujie M, Hahimoto D, et al. Increased serum kynurenine/tryptophan ratio correlates with disease progression in lung cancer. Lung Cancer. 2010; 67: 361-5.

21. Wang Y, Hu GF, Wang ZH. The status of immunosuppression in patients with stage IIIB or IV non-small-cell lung cancer correlates with the clinical characteristics and response to chemotherapy. Onco Targets Ther. 2017; 10: 3557-66.

22. Creelan BC, Antonia $S$, Bepler $G$, Garrett TI, Simon GR, Soliman HH Indoleamine 2,3-dioxygenase activity and clinical outcome following induction chemotherapy and concurrent chemoradiation in Stage III non-small cell lung cancer. Oncoimmunology. 2013; 2: e23428.

23. Wang W, Huang L, Jin JY, Jolly S, Zang Y, Wu H, et al. IDO Immune Status after Chemoradiation May Predict Survival in Lung Cancer Patients. Cancer Res. 2017.

24. Astigiano S, Morandi B, Costa R, Mastracci L, D'Agostino A, Ratto GB, et al. Eosinophil granulocytes account for indoleamine 2,3-dioxygenase-mediated immune escape in human non-small cell lung cancer. Neoplasia. 2005; 7: 390-6.

25. Rami-Porta R, Asamura H, Travis WD, Rusch VW. Lung cancer - major changes in the American Joint Committee on Cancer eighth edition cancer staging manual. CA Cancer J Clin. 2017; 67: 138-55.
26. Duan H, Zhang X, Wang FX, Cai MY, Ma GW, Yang H, et al. KIF-2C expression is correlated with poor prognosis of operable esophageal squamous cell carcinoma male patients. Oncotarget. 2016; 7: 80493-507.

27. Dahlin AM, Henriksson ML, Van Guelpen B, Stenling R, Oberg A, Rutegard J, et al. Colorectal cancer prognosis depends on T-cell infiltration and molecular characteristics of the tumor. Mod Pathol. 2011; 24: 671-82.

28. Brochez L, Chevolet I, Kruse V. The rationale of indoleamine 2,3-dioxygenase inhibition for cancer therapy. Eur J Cancer. 2017; 76: 167-82.

29. Heeren AM, van Dijk I, Berry D, Khelil M, Ferns D, Kole J, et al. Indoleamine 2,3-Dioxygenase Expression Pattern in the Tumor Microenvironment Predicts Clinical Outcome in Early Stage Cervical Cancer. Front Immunol. 2018; 9: 1598.

30. Engin A, Gonul II, Engin AB, Karamercan A, Sepici Dincel A, Dursun A. Relationship between indoleamine 2,3-dioxygenase activity and lymphatic invasion propensity of colorectal carcinoma. World J Gastroenterol. 2016; 22: 3592-601.

31. Godin-Ethier J, Pelletier S, Hanafi LA, Gannon PO, Forget MA, Routy JP, et al. Human activated T lymphocytes modulate IDO expression in tumors through Th1/Th2 balance. J Immunol. 2009; 183: 7752-60.

32. Schalper KA, Carvajal-Hausdorf D, McLaughlin J, Altan M, Velcheti V, Gaule $\mathrm{P}$, et al. Differential Expression and Significance of PD-L1, IDO-1, and B7-H4 in Human Lung Cancer. Clin Cancer Res. 2017; 23: 370-8.

33. Lob S, Konigsrainer A, Rammensee HG, Opelz G, Terness P. Inhibitors of indoleamine-2,3-dioxygenase for cancer therapy: can we see the wood for the trees? Nat Rev Cancer. 2009; 9: 445-52.

34. Ribas A, Wolchok JD. Cancer immunotherapy using checkpoint blockade. Science. 2018; 359: 1350-5.

35. Wang K, Huang Q, Qiu F, Sui M. Non-viral Delivery Systems for the Application in p53 Cancer Gene Therapy. Curr Med Chem. 2015; 22: 4118-36.

36. Borghaei H, Paz-Ares L, Horn L, Spigel DR, Steins M, Ready NE, et al. Nivolumab versus Docetaxel in Advanced Nonsquamous Non-Small-Cell Lung Cancer. N Engl J Med. 2015; 373: 1627-39. 